\title{
Análise comparativa de ferramentas de Montagem e Binning de metagenomas utilizando dados simulados microbianos
}

\author{
Rodrigo B. P. R. Pará1, Pedro H. D. M. Rocha ${ }^{1}$, Danielle C. C. Couto ${ }^{2}$, Renato R. M. \\ Oliveira $^{1}$, Regiane Kawasaki ${ }^{1}$ \\ ${ }^{1}$ Laboratório de Bioinformática e Computação de Alto Desempenho (LABIOCAD) - \\ Instituto de Ciências Exatas e Naturais - Universidade Federal do Pará (UFPA) \\ ${ }^{2}$ Laboratório Interdisciplinar em Tecnologias, Educação e Computação (LITEC) - \\ Campus Ananindeua - Universidade Federal do Pará (UFPA) \\ \{pedrohenriquedornele, renato.renison, rodrigopara\}@gmail.com, \\ \{danifc,kawasaki\} @ufpa.br
}

\begin{abstract}
Binning consists of grouping DNA sequences according to taxonomic units, widely used in the Metagenomics, field that studies the genome of communities of microorganisms. New tools are developed for metagenomic pipelines, necessitating the establishment of paradigms in this type of analysis through the verification of the performance of assembly and binning tools. For the comparative tests of this work, data sets of 10 and 100 species of bacteria were used, as well as 3 assembly softwares: IDBA_UD, Megahit and MetasSPAdes, and 2 binning softwares: MetaBAT-2.12.1 and MaxBin-2.2.4. We verified that MetaBAT exceeded MaxBin in the quality of the generated bins.
\end{abstract}

Resumo. Binning consiste em agrupar sequências de DNA de acordo com unidades taxonômicas, muito usado na Metagenômica, campo que estuda o genoma de comunidades de microrganismos. Novas ferramentas são desenvolvidas para pipelines metagenômicos, necessitando que se estabeleçam paradigmas neste tipo de análise através da verificação do desempenho de ferramentas de montagem e binning. Para os testes comparativos deste trabalho foram utilizados conjuntos de dados de 10 e 100 espécies de bactérias, além de 3 softwares montadores: IDBA_UD, Megahit e MetasSPAdes, e 2 softwares de binning: MetaBAT-2.12.1 e MaxBin-2.2.4. Verificou-se que o MetaBAT superou o MaxBin na qualidade dos bins gerados.

\section{Introdução}

A análise de dados metagenômicos é um processo mais complexo do que a genômica tradicional, devido a presença de muitos organismos na amostra, diferença de abundância entre estes organismos e sequências de DNA comum entre espécies [NAMIKI et al., 2012]. Sequenciar e montar os genomas destes organismos envolve fragmentar o DNA em pequenas sequências denominadas reads e posteriormente agrupá-las em sequências maiores (contigs) a fim de reconstruir o genoma original. 
O binning, um passo posterior à montagem, consiste em agrupar as sequências de DNA montadas de acordo com uma determinada unidade taxonômica (e.g. espécie) em arquivos denominados bins [WOOLEY et al., 2010]. Através do binning é possível obter informações de genomas ainda desconhecidos que são difíceis de identificar por outros métodos, e obter informações quanto aos tipos e quantidades de unidades taxonômicas presentes [SHARPTON, 2014].

A montagem de metagenomas é uma tarefa difícil e exige o desenvolvimento de novas técnicas e ferramentas, haja vista que os softwares de montagem de genomas tradicionais não são aptos a tratar dados metagenômicos [PENG et al., 2011].

Softwares de montagem de metagenomas foram desenvolvidas, como MetaSPades [NURK et al., 2012], IDBA-UD [PENG et al., 2012] e MetaVelvet [NAMIKI et al., 2012]. Entretanto, é necessário que se estabeleçam novos modelos de análise metagenômica através da verificação da eficácia das ferramentas de montagem em conjunto com o binning.

Este trabalho pretende comparar de maneira não enviesada o desempenho de ferramentas de montagem e binning de metagenomas. Muitos trabalhos relacionados a comparação de ferramentas são feitos no lançamento de uma ferramenta, com conjuntos de dados distintos, o que não permite uma comparação sólida. É interessante estabelecer um conjunto fixo de dados e a partir daí comparar o comportamento das ferramentas com estes dados. Para tal, o uso de dados simulados contendo genoma de espécies já conhecidas é recomendável pois permite aferir o desempenho destas ferramentas, através da comparação das sequências geradas com os genomas de referências dessas espécies. Essa análise poderá permitir a validação do pipeline e das ferramentas, permitindo sua aplicação em amostras com dados reais.

O presente artigo subdivide-se em mais três seções: Materiais e Métodos, abordando metodologia e ferramentas utilizadas no pipeline; Resultados e Discussões, contendo a análise comparativa dos resultados das ferramentas, e Conclusão, contendo considerações acerca da pesquisa e sugestões de trabalhos futuros.

\section{Materiais e Métodos}

Inicialmente foi realizada uma revisão bibliográfica dos trabalhos e ferramentas de Montagem e Binning comumente utilizados. Decidiu-se pelo MetaBAT-2.12.1 e MaxBin-2.2.4 devido a serem ferramentas atualizadas e com uma boa documentação disponível. Outras ferramentas, como o GroopM [IMELFORT et al., 2014] encontravam-se desatualizadas e por isso foram descartadas. Já o CONCOCT [ALNEBERG et al., 2014] recebeu uma atualização após a realização desta pesquisa em Outubro de 2018, portanto não foi incluído.

Para a realização dos testes comparativos, foram utilizados os conjuntos de dados de reads não tratadas de 10 e 100 espécies de bactérias de diferentes complexidades, retirados do artigo Assessment of metagenomic assembly using simulated next generation sequencing data [MENDE et al., 2012]. Estes dados foram gerados através do simulador de dados metagenômicos iMess (interactive MEtagenomic Simulation Software). Neste trabalho foram utilizadas as reads geradas pelo 
sequenciamento Illumina, que podem ser encontrados no link: www.bork.embl.de/ mende/simulated datal.

O tratamento de qualidade foi realizado pela ferramenta PRINSEQ [SCHMIEDER e EDWARDS, 2011]. Em seguida, as reads foram submetidas ao processo de montagem, realizado por três softwares montadores: IDBA_UD, Megahit, e MetasSPAdes, com valores default de k-mer (mínimo 20 e máximo 100 para o IDBA_UD; mínimo 21 e máximo 99 para o Megahit e 21, 33 e 55 para o MetaSPades). Em seguida, através da ferramenta Metaquast [MIKHEENKO; SAVELIEV; GUREVICH, 2016] foi feita a análise comparativa da qualidade das montagens, tendo como critérios cobertura do genoma e número de erros de montagem.

O melhor resultado obtido entre os três montadores foi submetido aos softwares de binning. Cada software de binning produz como saída um certo número de arquivos bins. Inicialmente as ferramentas foram rodadas com parâmetros default $\mathrm{e}$ posteriormente mudanças nos parâmetros foram feitas com o objetivo de observar o comportamento das ferramentas e a quantidade e qualidade dos bins gerados.

Para o dataset de 10 espécies, o MaxBin foi rodado uma única vez e o MetaBAT duas vezes: uma sem um arquivo de depth (arquivo que contém informação da abundância das espécies presentes na amostra; este arquivo é gerado separadamente) e posteriormente com o arquivo de depth.

Para o dataset de 100 espécies, o MaxBin foi rodado duas vezes, uma com os parâmetros default e outra com os parâmetros min_contig_length $=200$ e marker_set $=$ 40. Já o MetaBAT foi executado três vezes: a primeira execução com os parâmetros default; a segunda com os arquivos de depth e tamanho mínimo de bin de 130000, e a terceira com o arquivo de depth e tamanho mínimo de bin de 12000. Esses parâmetros foram escolhidos para aumentar o número de bins gerados na saída da ferramenta.

Cada um dos resultados gerados pelas ferramentas de binning foi novamente enviado ao Metaquast para avaliação. Através do Metaquast é possível observar a quantidade de bins gerados, qual o grau de completude de cada espécie e a quantidade total de alinhamento realizado dos contigs com os organismos da amostra.

Entretanto, somente analisar a quantidade de bins ou o grau de cobertura de cada bin alinhado ao genoma das espécies não é o suficiente para determinar a eficácia das ferramentas. Considerando-se que o resultado ideal seria ter todos os contigs de um bin pertencentes a uma única espécie, foi importante observar se ocorreram as seguintes situações: a) Um determinado bin alinhou com mais de uma espécie diferente; b) Dois ou mais bins fazem referência a uma mesma espécie;

Para analisar a eficácia de cada ferramenta ao categorizar os contigs de uma espécie por bin, foi criada uma Matriz de Confusão. Deseja-se saber se cada bin de fato está sendo mapeado aos contigs de uma única espécie.

Para isso, verifica-se todos os contigs presentes em um bin. A espécie com maior prevalência de contigs naquele bin será mapeada para àquele bin. A Tabela 1 exemplifica esse processo através das estatísticas obtidas do Metaquast para espécie Neisseria meningitidis MC58: 


\begin{tabular}{|c|c|c|}
\hline & Idba.MetaBAT3.3 & Idba.MetaBAT3.7 \\
\hline FRAÇÃO DE GENOMA & 0.187 & 77.107 \\
\hline MAIOR ALINHAMENTO & 4246 & 49978 \\
\hline ERROS DE MONTAGEM & 0 & 1 \\
\hline CONTIGS & 1 & 170 \\
\hline
\end{tabular}

Tabela 1 - Bins alinhados ao organismo Neisseria meningitidis MC58

Na parte inferior da Tabela 1, observa-se que o bin idbaMetaBAT3.7 possui 170 contigs alinhados à $N$. meningiditis MC58., enquanto idbaMETABAT3.3 possui somente 1. Logo, o bin idbaMetaBAT3.7 é mapeado para N. meningiditis MC58 por ter a maior correspondência de contigs. Após realizado o mapeamento individual de cada bin para cada organismo, cada contig presente no bin é classificado de acordo com uma das quatro situações presentes na Tabela 2:

\begin{tabular}{|c|l|l|}
\hline \multicolumn{1}{|c|}{ POSITIVO } & \multicolumn{1}{|c|}{ NEGATIVO } \\
\hline VERDADEIRO & $\begin{array}{l}\text { VERDADEIRO POSITIVO (VP): } \\
\text { Contig está no bin da espécie } x \text { e } \\
\text { pertence de fato à espécie } x .\end{array}$ & $\begin{array}{l}\text { VERDADEIRO NEGATIVO (VN): } \\
\text { Contig não está no bin da espécie } x \text { e não } \\
\text { pertence de fato à espécie } x\end{array}$ \\
\hline FALSO & $\begin{array}{l}\text { FALSO POSITIVO (FP): Contig está } \\
\text { no bin da espécie } x \text {, mas não pertence à } \\
\text { espécie } x .\end{array}$ & $\begin{array}{l}\text { FALSO NEGATIVO (FN): Contig não } \\
\text { está no bin da espécie } x \text {, mas pertence à } \\
\text { espécie } x .\end{array}$ \\
\hline
\end{tabular}

Tabela 2 - Matriz de confusão de contigs presentes nos bins

A Matriz de Confusão foi calculada individualmente para cada bin. Em seguida, cada bin foi avaliado segundo as seguintes métricas:

Precisão: indica se há presença de contaminantes (contigs de outras espécies) em um bin. Quantos contigs presentes em um bin são da espécie desejada? Fórmula: $P R E C I S \tilde{A O}=V P /(V P+F P)$

Sensibilidade: Frequência que a ferramenta é capaz de colocar no mesmo bin os contigs de uma espécie. Considerando todos os contigs de uma espécie, quantos deles foram classificados no bin correto? Fórmula: SENSIBILIDADE $=V P /(V P+F N)$

Especificidade: calcula a frequência que a ferramenta é capaz de colocar em outros bins os contigs que não pertencem à espécie analisada. Dos contigs que não pertencem a espécie analisada, quantos deles realmente foram categorizados em outros bins? Fórmula: ESPECIFICIDADE $=V N /(V N+F P)$

Acurácia: indica no geral o quão correta foi feita a classificação pela ferramenta. Ela é calculada através da soma dos Verdadeiros Positivos e Verdadeiros Negativos, dividido pelo total. Fórmula: $A C U R A ́ C I A=(V P+V N) /(V P+F P+V N+F N)$ 
Para obter o desempenho geral de cada ferramenta, calculou-se a média das métricas individuais dos bins. O pipeline utilizado na análise de metagenomas está descrito na Figura 8:

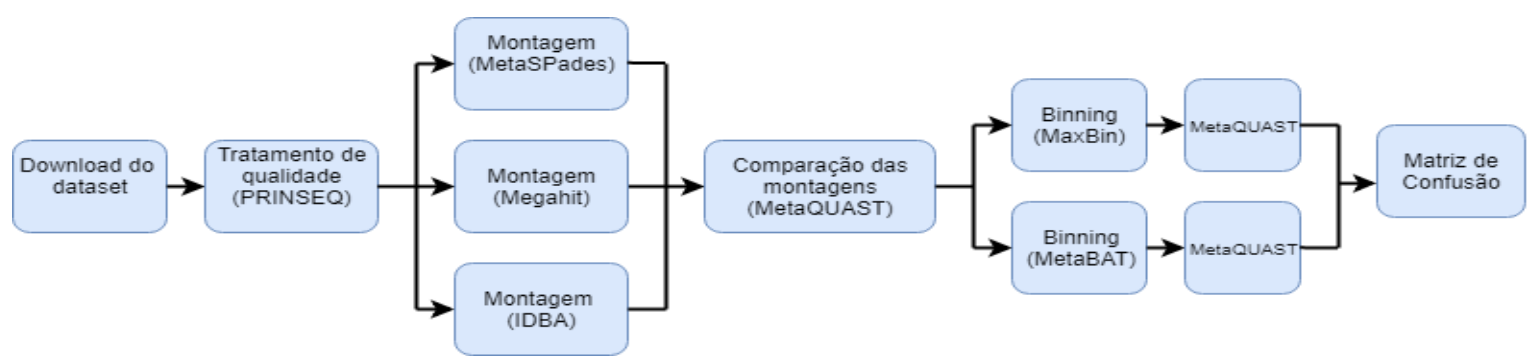

Figura 8 - Pipeline para comparação de ferramentas de montagem e binning

\section{Resultados e Discussões}

Os arquivos de bins gerados seguem a padronização nome do montador seguido pelo nome da ferramenta de binning. Diferentes execuções das mesmas ferramentas são indicadas por números (e.g. idbaMetaBAT1, idbaMetaBAT2 etc.).

\subsection{Dataset de 10 Espécies}

Para a montagem de 10 espécies, o IDBA foi o que obteve melhor cobertura de genoma (99.3\%), como mostra o Quadro 1:

\begin{tabular}{|l|l|l|l|}
\hline & IDBA_contig & Megahit_contig & Spades_contig \\
\hline FRAÇÃO DE GENOMA & 99.314 & 99.108 & 99.051 \\
\hline MAIOR ALINHAMENTO & 1190435 & 1100460 & 633416 \\
\hline ERROS DE MONTAGEM & 6 & 25 & 13 \\
\hline CONTIGS & 837 & 447 & 817 \\
\hline
\end{tabular}

Quadro 1 - Comparação dos montadores para dataset de 10 espécies

Em seguida, o binning foi executado pelo MetaBAT e MaxBin. A quantidade de bins gerados e os parâmetros utilizados podem ser vistos no Quadro 2:

\begin{tabular}{|l|l|l|l|}
\hline & IdbaMaxBin & IdbaMetaBAT1 & IdbaMetaBAT2 \\
\hline BINS & 10 & 11 & 10 \\
\hline PARÂMETROS & default & default, sem arquivo de depth & default, com arquivo de depth \\
\hline
\end{tabular}

Quadro 2 - Quantidade de bins gerados para dataset de 10 Espécies 
No dataset de 10 espécies, o MaxBin foi capaz de gerar 10 arquivos fasta separados. Para rodar, o MaxBin necessita obrigatoriamente de um arquivo contendo a abundância dos organismos da amostra ou de um arquivo contendo as reads sequenciadas. Para esta ferramenta, foi utilizado o arquivo de 10 espécies contendo as reads sequenciadas. Através do arquivo de reads, o MaxBin é capaz de gerar o arquivo de abundância das espécies utilizando o BowTie2 [LANGMEAD; SALZBERG, 2012]. O MetaBAT por sua vez obteve um resultado mais preciso quando o arquivo de depth foi utilizado, gerando 10 bins.

\subsection{Dataset de 100 Espécies}

Para o conjunto de 100 espécies, os montadores Megahit e MetaSPades cobriram uma fração semelhante do genoma da amostra (aproximadamente 62\%). Entretanto, o Megahit obteve uma quantidade menor de erros de montagem (2766), sobressaindo-se ao MetaSPades (8925), conforme mostra o Quadro 3:

\begin{tabular}{|c|c|c|c|}
\hline & IDBA_contig & Megahit_final_contig & Spades_contig \\
\hline FRAÇÃO DE GENOMA & 50.912 & 62.717 & 62.873 \\
\hline MAIOR ALINHAMENTO & 133685 & 195515 & 144343 \\
\hline ERROS DE MONTAGEM & 64507 & 2766 & 8925 \\
\hline CONTIGS & 814833037 & 185742 & 183858 \\
\hline
\end{tabular}

Quadro 3 - Comparação dos montadores para dataset de 100 espécies

De forma análoga aos resultados de 10 espécies, o Quadro 4 representa a quantidade de bins gerados e os parâmetros utilizados para cada uma das execuções do MaxBin e MetaBAT, utilizando como entrada os contigs gerados pelo Megahit:

\begin{tabular}{|c|c|c|c|c|c|}
\hline & Megahit & Megahit & Megahit & Megahit & Megahit \\
MaxBin1 & MaxBin2 & MetaBAT1 & MetaBAT2 & MetaBAT3 \\
\hline BINS & 33 & 76 & 15 & 24 & 96 \\
\hline $\begin{array}{c}\text { PARÂMET } \\
\text { ROS }\end{array}$ & Default & $\begin{array}{c}\text { min_contig_length } \\
=200 ; \\
\text { markerset }=40\end{array}$ & $\begin{array}{c}\text { Default, } \\
\text { com arquivo de } \\
\text { depth }\end{array}$ & $\begin{array}{c}\text { som arquivo de } \\
\text { depth }\end{array}$ & $\begin{array}{c}\text { com arquivo } \\
\text { de depth }\end{array}$ \\
\hline
\end{tabular}

Quadro 4 - Quantidade de bins gerados para dataset de 100 Espécies

Com parâmetros padrão, O MaxBin gerou somente 33 bins, uma quantidade baixa considerando o tamanho da amostra. Na segunda execução, utilizou-se os parâmetros min_contig_length e markerset setados para 200 e 40, respectivamente. Para chegar nesses valores, verificou-se através da ferramenta PRINSEQ que o menor contig gerado pelo Megahit possuía tamanho de 200 pares de base. Definiu-se então como 200 o tamanho mínimo de contig.

Já o parâmetro markerset representa os marcadores genéticos presentes na maioria das bactérias e é definido em 107 como default. Uma outra opção é utilizar o 
markerset com valor 40, o que tende a dividir a saída em um número maior de bins. Com esses parâmetros, o MaxBin gerou 76 bins.

O MetaBAT não permite que o tamanho mínimo do contig seja configurado para 200. O tamanho mínimo do parâmetro de menor contig para realizar o bin é de 1500 [KANG et al., 2015]. Devido a isso, outros parâmetros foram utilizados.

No primeiro teste com parâmetros padrão (com tamanho mínimo de bin igual a 200000), o MetaBAT retornou como saída somente 15 arquivos de bin (MegahitMetaBAT1). Para o segundo teste, modificou-se o parâmetro -s que representa o tamanho mínimo de bin gerado. Para decidir um tamanho razoável de bin, verificou-se que entre os bins gerados pelo MaxBin, o menor equivalia a 130000 pares de base. Esse valor foi utilizado como parâmetro no MetaBAT, gerando 24 bins (MegahitMetaBAT2). Testes posteriores foram realizados com valores menores de bin para tentar aproximar a quantidade de bins à quantidade de organismos, chegando-se ao número de 12000 pares de base como tamanho mínimo. Com esse valor, 96 bins foram gerados pelo MetaBAT (MegahitMetaBAT3).

\subsection{Matriz de Confusão (10 espécies)}

O Quadro 5 representa a média das métricas da Matriz de Confusão para as melhores execuções das ferramentas de binning (aquelas cuja quantidade de bins gerados se aproxima da quantidade de organismos na amostra):

\begin{tabular}{|c|c|c|}
\hline & idbaMetaBAT2 & idbaMaxBin \\
\hline ESPECIFICIDADE & $99,72 \%$ & $97,99 \%$ \\
\hline SENSIBILIDADE & $99,52 \%$ & $84,25 \%$ \\
\hline ACURÁCIA & $99,71 \%$ & $96,63 \%$ \\
\hline PRECISÃO & $94,34 \%$ & $75,14 \%$ \\
\hline
\end{tabular}

Quadro 5 - Média das métricas para idbaMetaBAT2 e MaxBin

O MetaBAT obteve melhores valores em todas as métricas comparado ao MaxBin, obtendo acima de 99\% em Especificidade, Sensibilidade e Acurácia, e acima de 94\% em Precisão. O MaxBin obteve um nível de precisão de 75,14\%. Isso significa que há uma quantidade maior de contigs contaminantes nos bins gerados pelo MaxBin.

\subsection{Matriz de Confusão (100 espécies)}

Para o dataset de 100 espécies, a Matriz de Confusão foi criada para as execuções MegahitMaxBin2 e MegahitMetaBAT3. O Quadro 6 compara os resultados:

\begin{tabular}{|c|c|c|}
\hline & MegahitMetaBAT3 & MegahitMaxBin2 \\
\hline ESPECIFICIDADE & $99,75 \%$ & $99,13 \%$ \\
\hline SENSIBILIDADE & $39,80 \%$ & $35,50 \%$ \\
\hline ACURÁCIA & $98,32 \%$ & $98,08 \%$ \\
\hline PRECISÃO & $80,73 \%$ & $37,47 \%$ \\
\hline
\end{tabular}


O MetaBAT alcançou melhores resultados que o MaxBin em todas as métricas. Entretanto, ambos tiveram baixa sensibilidade, indicando que os contigs de uma espécie se encontram dispersos por vários bins.

Tanto o dataset de 10 quanto o de 100 espécies foram gerados com a mesma quantidade de reads [MENDE et al., 2012], o que fez com que o dataset de 100 espécies tivesse baixa cobertura dos genomas, impactando na montagem e no binning.

\section{Conclusão}

O MetaBAT se sobressaiu ao MaxBin na qualidade dos bins gerados, tanto para o dataset de 10 espécies quanto para o de 100 espécies; e o arquivo contendo os dados sobre a abundância dos contigs é necessário para que as ferramentas de binning retornem um resultado mais preciso. Além disso, a cobertura das reads sequenciadas possui um impacto direto na qualidade da montagem e do binning.

Futuramente, é interessante que novos testes sejam realizados com outras ferramentas de binning, como o CONCOCT, e seu desempenho comparado com as ferramentas presentes neste estudo. Testes posteriores utilizando um valor maior de cobertura para o dataset de 100 espécies também se fazem necessários.

\section{Referências}

IMELFORT, M. et al. GroopM: an automated tool for the recovery of population genomes from related metagenomes. PeerJ, v. 2, p. e603, 2014.

KANG, D. D. et al. MetaBAT, an efficient tool for accurately reconstructing single genomes from complex microbial communities. PeerJ, v. 3, p. e1165, 2015.

LANGMEAD, B.; SALZBERG, S. L. Fast gapped-read alignment with Bowtie 2. Nature Methods, v. 9, n.4, p. 357-359, 2012.

MENDE, D. R. et al. Assessment of Metagenomic Assembly Using Simulated Next Generation Sequencing Data. PLos ONE v. 7, n. 2, 2012.

MIKHEENKO, A.; SAVELIEV, V.; GUREVICH, A. MetaQUAST: Evaluation of metagenome assemblies. Bioinformatics, v. 32, n. 7, 2016.

NAMIKI, T. et al. MetaVelvet: an extension of Velvet assembler to de novo metagenome assembly from short sequence reads. Nucleic Acids Research, v. 40, n. 20, 2012.

NURK, S et al. MetaSPades: a new versatile metagenomics assembler Sergey. Genome Research, v. 27, n. 5, p. 824-834, 2017.

PENG, Y. et al. Meta-IDBA: a de Novo assembler for metagenomic data. Bioinformatics, v. 27, p. 94-101, 2011.

SCHMIEDER, R.; EDWARDS, R. Quality control and preprocessing of metagenomic datasets. Bioinformatics, v. 27, n. 6, p. 863-864, 2011.

SHARPTON, T. J. An introduction to the analysis of shotgun metagenomic data. Frontiers in Plant Science, v. 5, p. 1-14, 2014.

WOOley, J. C.; GODZIK, A.; FRIEDBERG, I. A Primer on Metagenomics. PLoS Computational Biology, v. 6, n. 2, 2010. 